\title{
Determination of the Molar Extinction Coefficient of Colloidal Selenium for Optical Characterization of Stabilized Nanoparticulate Dispersions
}

\author{
Matthias Schuster*, Tobias Wernicke, Stefan A Möckel and Peter J Wellmann
}

\author{
Department of Materials Science 6, Friedrich-Alexander-University Erlangen-Nürnberg, Germany
}

\begin{abstract}
To prepare fully printed copper-indium-diselenide solar-cell-absorbers it is necessary to have well defined and printable precursor-inks. In this work, the synthesis and different approaches for the colloidal stabilization of Selenium (Se) nanoparticles are presented, which should ultimately serve as a selenium source for $\mathrm{ClSe}$ absorbers. The produced particles were stabilized with different concentrations of Polyvinylpyrrolidone (PVP) and micelles (TX100) respectively. The resulting suspensions were analyzed in aspect of particle size and colloidal stability using dynamic light scattering (DLS). It was shown, that both approaches lead to stable colloidal suspensions with ca. $80-100 \mathrm{~nm}$ particle size. The PVP stabilized suspension stays stable for four weeks at a Zeta potential of $>35-25 \mathrm{mV}$ whereas the TX100 stabilized suspension was only short-time-stable due to loss in Zeta potential over time. UV/VIS spectroscopy and Lambert-Beer's law were used to determine the concentration of $\mathrm{Se}$ in the dispersion. The molar extinction coefficient $\varepsilon$ of colloidal selenium was calculated to be $1.44 \times 10^{3} \mathrm{l} /(\mathrm{mol} \cdot \mathrm{cm})$ for $\varepsilon(\mathrm{Se})$ respectively $2.63 \times 10^{7} \mathrm{l} /(\mathrm{mol} \cdot \mathrm{cm})$ for $\varepsilon($ Se-NP) $\pm 9 \%$ in both cases.
\end{abstract}

\section{Keywords}

Printable nanoparticles, Colloidal selenium, Stabilization, Dynamic light scattering, Concentration, Molar extinction coefficient, UV/VIS

\section{Highlights}

- Evaluation of PVP vs. TX100 micelles as particle stabilizer

- Determination of Se concentration in dispersion via UV/VIS spectroscopy

- Determination of molar extinction coefficient of colloidal Selenium

\section{Introduction}

$\mathrm{CuInSe}_{2}$ (CISe) materials have become very attractive as absorber layer for thin film solar cells, due to a suitable band gap, a high extinction coefficient and good materials stability [1-5]. Different approaches to produce CISe via co-evaporation, pulsed Laser deposition or sputtering have been reported [6-8]. Due to process complexity and the problem of scaling up the process various nonvacuum approaches have been suggested: the Selenium integrated by annealing a $\mathrm{Cu}$-In-precursor under $\mathrm{H}_{2} \mathrm{Se}$ and $\mathrm{N}_{2}$ atmosphere at a temperature of $\sim 450^{\circ} \mathrm{C}$ [9]. This procedure holds highly toxic $\mathrm{H}_{2} \mathrm{Se}$ and is potentially explosive, due to free hydrogen. Therefore the preparation of a solid Se film via sputtering, electrochemical deposition or evaporation has been considered [10-14]. So far, these approaches lead to a poor intermixture of Se and Cu-In [15]. Recent research was conducted on all-printable precursors using bimetallic $\mathrm{Cu}$-In nanoparticles [16]. Such precursors may be mixed and subsequently printed, leading to a well distributed $(\mathrm{Cu}+\mathrm{In})$ :Se ratio. A method to precisely monitor and tune the molar composition of the dispersion and in particular the Se content would be beneficial.

In the present work the optical transmission of Se dispersions in combination with Lambert-Beer's law is used to determine the concentration of Se in the dispersions. To apply the Lambert-Beer's law accurately, knowledge about the molar extinction coefficient is necessary. However, hardly any data are available in literature for nanoparticulate Se dispersions. To determine the molar extinction coefficient of colloidal Se we have prepared a series of $\mathrm{Cu}-\mathrm{In}+\mathrm{Se}$ dispersions with varying Se concentrations. To determine the $(\mathrm{Cu}+\mathrm{In}): \mathrm{Se}$ ratio, layers were deposited and characterized using energy dispersive X-ray spectroscopy (EDX). In parallel optical transmission/extinction of the Se dispersion was determined by UV/ VIS spectroscopy. Using Lambert-Beer's law the molar extinction coefficient was deduced from the experimentally determined Se concentration and optical absorbance.

A commonly used synthesis route of Se nanoparticles is to

*Corresponding author: Matthias Schuster, Department of Materials Science 6, Materials for Electronics and Energy Technology, Friedrich-Alexander-University Erlangen-Nürnberg, Martensstr. 7, 91058 Erlangen, Germany, E-mail: msc.matthias.schuster@fau.de

Received: March 01, 2016: Accepted: April 11, 2016: Published: April 13, 2016

Copyright: (c) 2016 Schuster M, et al. This is an open-access article distributed under the terms of the Creative Commons Attribution License, which permits unrestricted use, distribution, and reproduction in any medium, provided the original author and source are credited. 


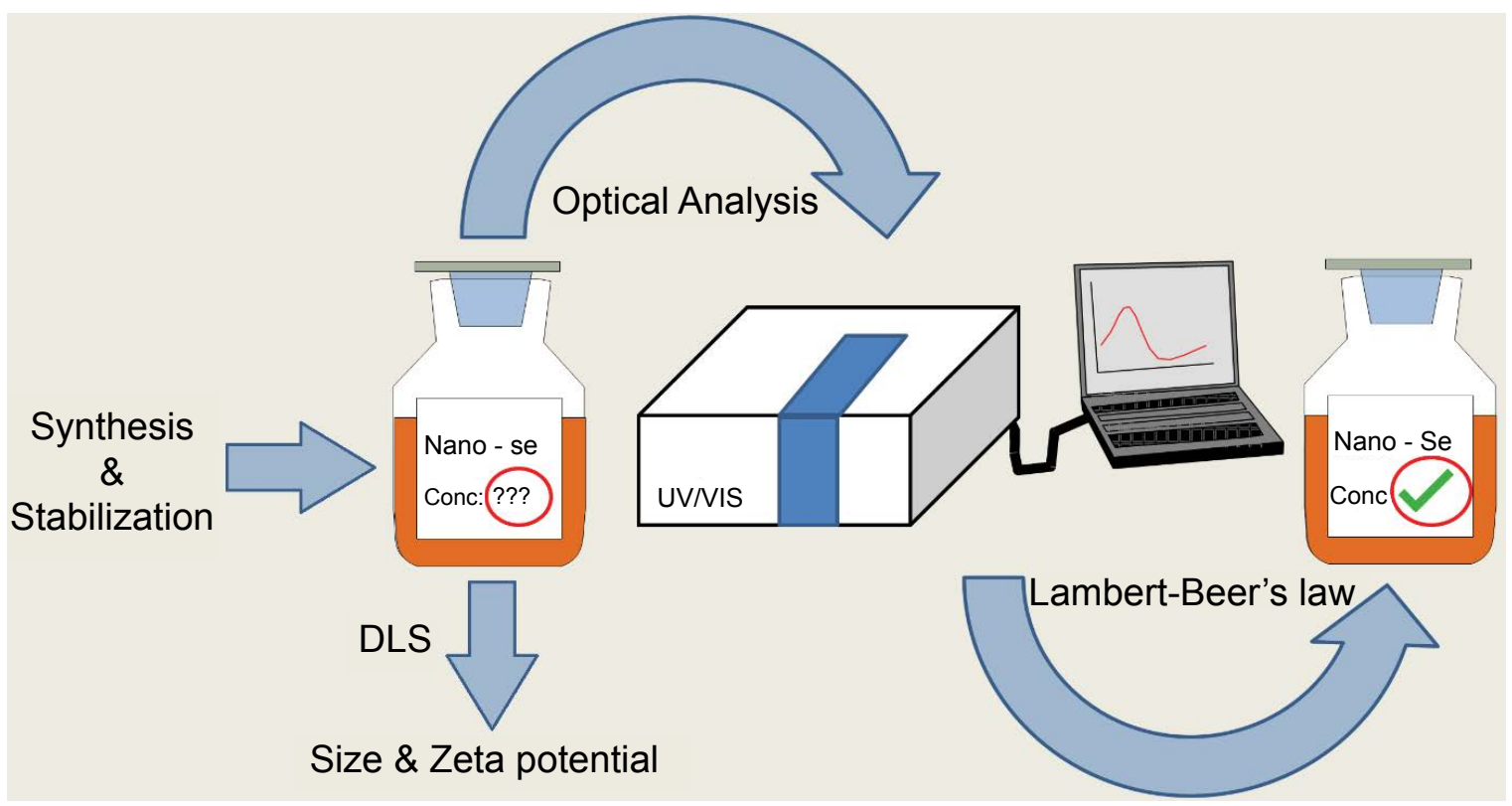

Figure 1: Scheme showing the experimental process from synthesis and stabilization of Se nanoparticles with unknown concentration to the characterization with DLS and UV/VIS to achieve size, Zeta potential and concentration.

precipitate Se-NP at room temperature and ambient pressure conditions from a $\mathrm{Na}_{2} \mathrm{SeSO}_{3}$ solution by adding $\mathrm{HCl}[17,18]$. Various approaches to stabilize Se nanoparticles have been investigated to prevent agglomeration [19-21]. In this work, the stabilization of chemically synthesized Se-NP is achieved by using Polyvinylpyrrolidone (PVP) and Triton x100 Micelles (TX100) respectively. The concentrations of PVP and TX100 were varied for different syntheses and the resulting particles were analyzed using dynamic light scattering (DLS) for mean particle size and Zeta potential measurements. Using the above mentioned UV/ VIS spectroscopy and Lambert-Beer's law to determine the Se concentration a relatively simple, time saving and reliable method was implemented in the deposition process of well defined, fully printable Se precursors. A scheme of the desired experimental process can be seen in figure 1.

\section{Experimental}

$\mathrm{Na}_{2} \mathrm{SO}_{3}$ ( $\geq 98.0 \%$, dehydrated, Sigma Aldrich), Se powder ( $\geq 99.5 \%$, Sigma Aldrich), Polyvinylpyrrolidone (PVP, $10000 \mathrm{~g} /$ mol, Fluka Analytical), Polyethylene-glycol-tert-octylphenyl-ether micelles (TX100) (Triton x100, laboratory grade, Sigma Aldrich), Hydrochloric acid ( $\mathrm{HCl}$ ) (Merck, 37\% fuming) and Trisodiumcitrate (Merck, dehydrated) were used as received without further purification for the experiments. $\mathrm{Cu}$-In particles for the determination of Se concentration were synthesized at our institute as described by Schuster et al. [22].

\section{Se nanoparticle synthesis}

The nanoparticles were fabricated from a $0.127 \mathrm{~mol} / \mathrm{l} \mathrm{Na} \mathrm{SeSO}_{3}$ solution [17]. This solution was prepared by dissolving $15.0 \mathrm{~g}$ of $\mathrm{Na}_{2} \mathrm{SO}_{3}$ in $200 \mathrm{ml}$ of deionized water at a temperature of $80^{\circ} \mathrm{C}$ under constant stirring. To prevent evaporation of the solvent, a water cooled reflux condenser was attached. Once the solution was clear $2.0 \mathrm{~g}$ of Se powder were added. Referring to Stroyuk et al. [18] the following reaction takes place forming $\mathrm{Na}_{2} \mathrm{SeSO}_{3}$.

$$
\mathrm{Na}_{2} \mathrm{SO}_{3}+\mathrm{Se} \rightarrow \mathrm{Na}_{2} \mathrm{SeSO}_{3}
$$

The solution was stirred at $80^{\circ} \mathrm{C}$ for 24 hours before being used as storage-batch-solution for the nanoparticle synthesis itself.

Several syntheses were carried out using different concentrations of PVP and TX100 respectively. Concentrations of $0.675-2.7 \mathrm{mmol} / \mathrm{l}$ for PVP and $2-63.3 \mathrm{mmol} / \mathrm{l}$ for the TX100 were implemented. For the syntheses with PVP, 0.306 - 1.225 g trisodium-citrate was added as an acidic buffer. For all syntheses, the respective stabilizer was first dissolved in $65.0 \mathrm{ml}$ deionized water for PVP or in $25.0 \mathrm{ml}$ for TX100so fewer micelles achieve the desired concentrations. AVWR pH 100 mobile $\mathrm{pH}$-meter with temperature sensor was attached to measure the synthesis parameters. After completely dissolving the stabilizing agent, $25.0 \mathrm{ml}$ of the previously prepared $80^{\circ} \mathrm{C}$ warm $\mathrm{Na}_{2} \mathrm{SeSO}_{3}$ storage-batch-solution was added to the reaction flask, raising the $\mathrm{pH}$ to $\sim 10$ and the temperature significantly above room temperature. The reaction solution was then kept stirring until it cooled down to room temperature naturally. To initialize the Se nanoparticle precipitation, $\mathrm{HCl}$ was added successively in $5 \mathrm{ml}$ steps until the $\mathrm{pH}$ reached a value of $\leq 0.5$. The solution turned from clear to an orange-red murky solution in every performed synthesis. The solution was stirred for 1 hour after which the $\mathrm{pH}$ reached ca. -0.05 . Subsequently, the solution was centrifuged at $12500 \mathrm{rpm}$ for 20 minutes at $10^{\circ} \mathrm{C}$ to extract the nanoparticles. After washing the Se-NP with $100 \mathrm{ml}$ deionized water, thus re dispersing them, the dispersion was neutralized using the $\mathrm{pH}$ meter and $1 \mathrm{M} \mathrm{NaOH}$ solution and centrifuged again. For further experiments and the application as printable Se precursor for thinfilm CISe solar-cells the nanoparticles were concentrated and stored in dispersion. For the characterization of the nanoparticles $30 \mu \mathrm{l}$ of the particle solution was placed in a $10 \times 10 \mathrm{~mm}^{2}$ cuvette and diluted with $3 \mathrm{ml}$ of deionized water.

\section{Se nanoparticle characterization}

The size, size distribution and Zeta potential of the particles in the diluted dispersions were determined by Dynamic Light scattering (DLS) using a MALVERN Zetasizer Nano ZS. Differently stabilized samples were measured over a time period of 4 weeks storage to observe aging stability of both stabilizers.

To determine the concentration $\mathrm{c}$ of Se in the syntheses, $20.0 \mathrm{ml}$ of a Se dispersion was mixed with $100.00 \mathrm{mg} \mathrm{Cu}$-In Nanoparticles, and analyzed using energy dispersive X-ray spectroscopy (EDX) with an accelerating voltage of $20 \mathrm{kV}$ in a Jeol JSM 6400 Scanning Electron Microscope.

The transmission of freshly synthesized, PVP-stabilized 
dispersions was measured between $350 \mathrm{~nm}$ and $750 \mathrm{~nm}$ using a Perkin Elmer Lambda 950 UV/VIS spectrometer. For the measurement $30 \mu \mathrm{l}$ of the respective Se nanoparticle dispersion was placed in a $10 \times$ $10 \mathrm{~mm}^{2}$ cuvette and diluted with $3 \mathrm{ml}$ of deionized water. To reduce scattering effects and to use the measurements as a potential cross reference to determine the Se concentration, the measurement was conducted in an integrating sphere.

For the determination of particle morphology scanning electron microscopy (SEM)-images were taken, using a Zeiss FESEM ultra 55 with an acceleration voltage of $5 \mathrm{kV}$.

\section{Results and Discussion}

\section{Particle stabilization}

After several preliminary tests to optimize the different stabilizer concentrations, the characterizations were conducted on samples prepared with $1.35 \mathrm{mmol} / \mathrm{l}$ PVP or $63.3 \mathrm{mmol} / \mathrm{l} \mathrm{TX100.} \mathrm{Other} \mathrm{samples}$ with lower concentrations agglomerated within a very short time and therefore were not investigated. To control colloidal stability the Zeta potential was measured over a time of 4 weeks (Figure 2).

The Zeta potential stays below $-30 \mathrm{mV}$ during the first week of

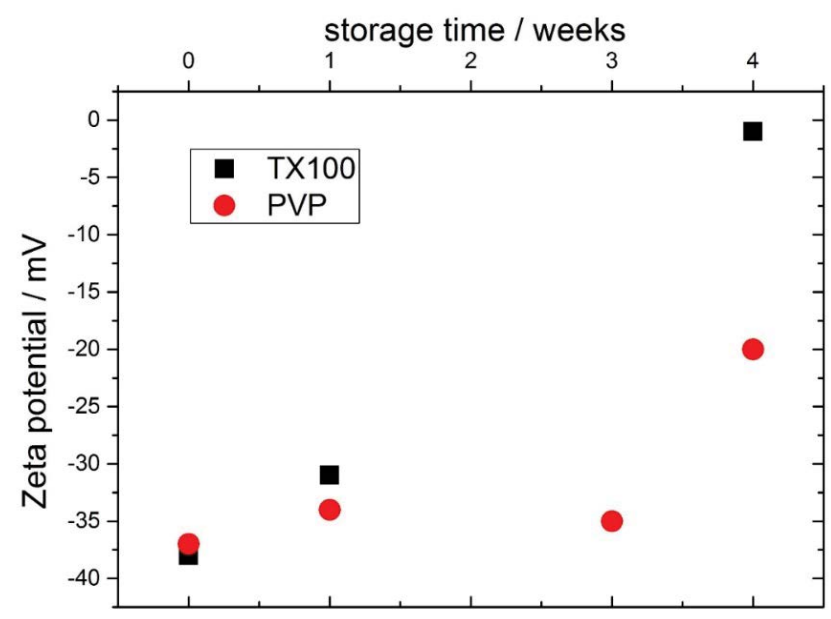

Figure 2: The Zeta potential and correlating to that the colloidal stability of samples with PVP and TX100 over a time of 4 weeks. The Zeta potential of the TX100 micelles decreases to almost 0 , whereas the Zeta potential of the PVP only decreases slightly after 4 weeks of storage. storage. After four weeks, the TX100 stabilized particles agglomerate and do not remain colloidal because of the Zeta potential decrease to $-1.6 \mathrm{mV}$. The PVP stabilized particles however remain stable which is reflected by a Zeta potential of $-20 \mathrm{mV}$ after four weeks of storage.

This could be explained by the stabilization principle of the respective stabilizer. Polymers, such as PVP, attach themselves to the particles [23] leading to a permanent chemically bound hull. Micelles however form an electrostatic cage around the particles due to a energetically favorable balance between enthalpy and entropy [24]. The Se particles however seem to be able to migrate through the micelle membrane leading to particle growth. Therefore this stabilization principle is apparently not convenient for Se nanoparticles over a longer period of time since the tendency of particle growth and agglomeration seems to be stronger than the electrostatic shielding of the micelles. In conclusion, the protecting layer of PVP seems to be much more impermeable, which is supported by the relating growth mechanisms shown by Shin et al. [25]. Therefore the following experiments to find out the molar extinction coefficient of colloidal selenium was performed with PVP stabilized dispersions.

\section{Particle size}

The particle size was determined with DLS by measuring the prepared samples over a period of four weeks (Figure 3). The freshly prepared and immediately measured PVP stabilized particles had size of $\sim 80 \mathrm{~nm}$ with an obvious size distribution between 30 and $110 \mathrm{~nm}$. All other PVP stabilized particles had a size of $\sim 100 \mathrm{~nm}$ and a much smaller size distribution. The micelle stabilized particles also had a size of $\sim 100 \mathrm{~nm}$ when measured freshly after preparation and after 1 week of storage. After 4 weeks of storage however, they showed an increase of size to $\sim 450 \mathrm{~nm}$.

Apparently, the PVP stabilized particles are much smaller at the beginning of the precipitation and grow until the equilibrium between growth tendency and stabilization is reached. The growth mechanisms of PVP stabilized particles has been well investigated by different research groups $[23,25]$. The reason why the micelles do not show a size shift after being freshly synthesized could be explained with the critical micelle concentration (cmc) [24]. The used concentrations are all above the $\mathrm{cmc}$ which means, the shielding hull of the micelles have already formed and the particles can grow within them.

To confirm these results and to show the morphology of the synthesized particles, SEM pictures were taken. Particles stabilized with PVP (Figure 4) are spherical and fairly distributed particles,
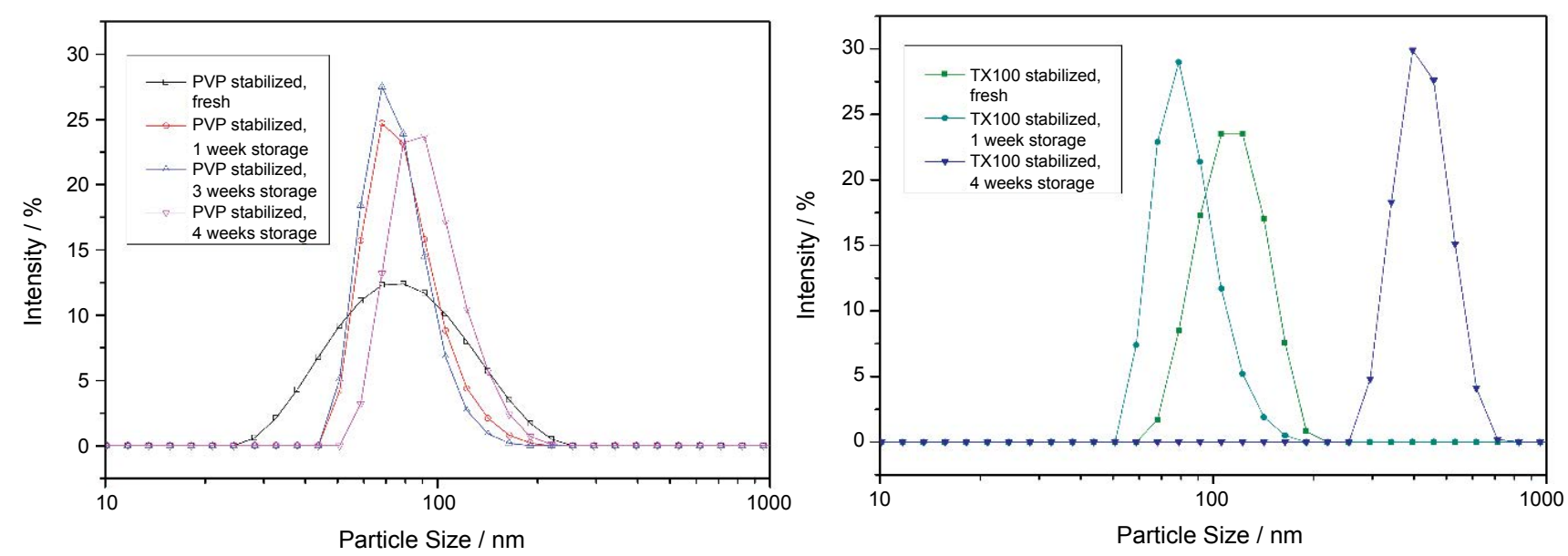

Figure 3: Particle size changes over four weeks of PVP (left) and of TX100-micelle (right) stabilized Se particles: The micelle stabilized particles grow after 4 weeks of storage time, while the size of PVP stabilized particles stays constant over the same time. Fresh PVP stabilized particles show a broad size distribution. 


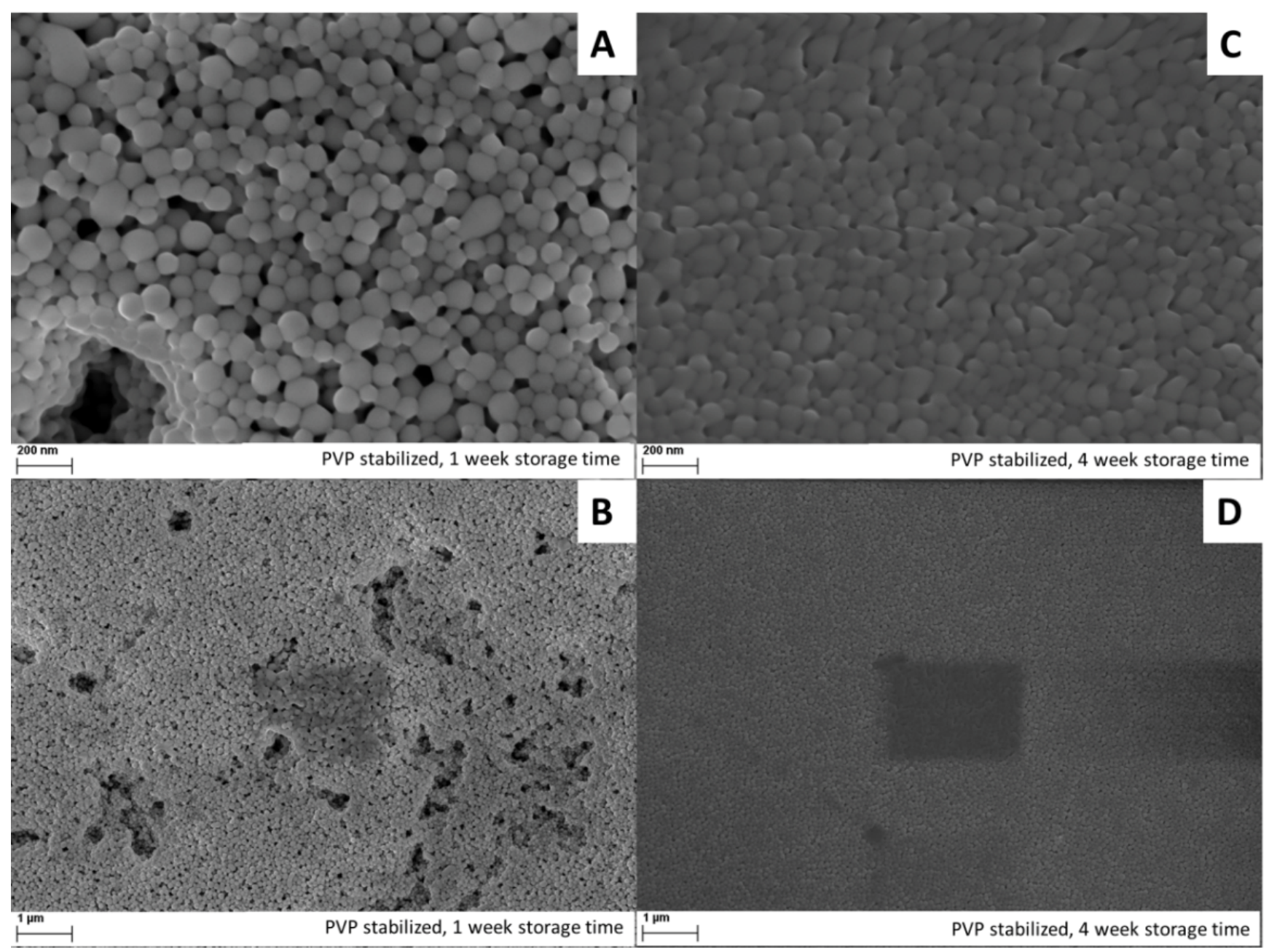

Figure 4: SEM pictures of Se particles, stabilized with PVP after 1 week $(A, B)$ and 4 weeks $(C, D)$ of storage. In the pictures $B$ and D, a footprint of sintermarks can be seen where the picture with higher magnification was taken.

Table 1: Atomic ratios of different Se synthesis mixed with Cu-In nanoparticles measured via EDX.

\begin{tabular}{|l|l|l|l|l|}
\hline Sample no. & Mean ratio $\mathrm{Cu}$ in At.\% & Mean ratio In in At.\% & Mean ratio Se in At.\% & Calculated Se yield in mg \\
\hline 1 & 31.50 & 30.70 & 37.74 & 140.22 \\
\hline 2 & 33.24 & 37.09 & 29.66 & 89.13 \\
\hline 3 & 32.07 & 39.00 & 28.93 & 79.82 \\
\hline 4 & 31.22 & 36.48 & 32.30 & 99.65 \\
\hline
\end{tabular}

which maintain this shape even after 4 weeks of storage time. However, the particles are rather sensitive concerning the stability of their shape. When applying higher acceleration voltages, the particles start to sinter. This effect intensifies with longer storage time. This is attributed to a decrease in particle stability over time, most likely due to particle ripening.

The same effect can be observed for the TX100 stabilized particles (Figure 5) but with an even higher tendency to sinter under the SEM. However, the structure was more spattered and the agglomeration/ ripening process was much faster which can be seen after 4 weeks of storage. The particles are clustered to big $\mu \mathrm{m}$ sized structures and even form large needle like structures that start to become visible after the first week of storage. This can be explained by the assumed loss of colloidal stability due to the lack of stabilization potential of the micelles.

\section{Optical properties}

Due to their higher colloidal stability and application potential, only the PVP stabilized Se particles were evaluated concerning their optical properties. The objective was to look for a correlation between UV/VIS extinction behavior and particle concentration. According to the Lambert-Beer-Law the extinction $\mathrm{E}$ is defined as:

$$
E=-\log T=\varepsilon * c * d
$$

$\mathrm{T}$ being the measured transmission, $\varepsilon$ the molar extinction coefficient, $\mathrm{c}$ the concentration of Se and $\mathrm{d}$ the path length which the light travels through the material, with our measurement setup $10 \mathrm{~mm}$. To our best knowledge and literature search, there are no values for the molar extinction coefficient $\varepsilon$ for colloidal Se available so far. By rearranging the Lambert-Beer's law it can provide $\varepsilon$, if the concentration is given:

$$
\varepsilon=\frac{-\log T}{c * d}
$$

To determine the concentration $\mathrm{c}$, a mixture of $\mathrm{Se}$ and $\mathrm{Cu}$ In nanoparticles was prepared and analyzed using EDX. Since we used well defined amount of $\mathrm{Cu}$-In, it can be used as a reference to determine the Se amount in the mixture. Table 1 shows the atomic ratios of four samples. 


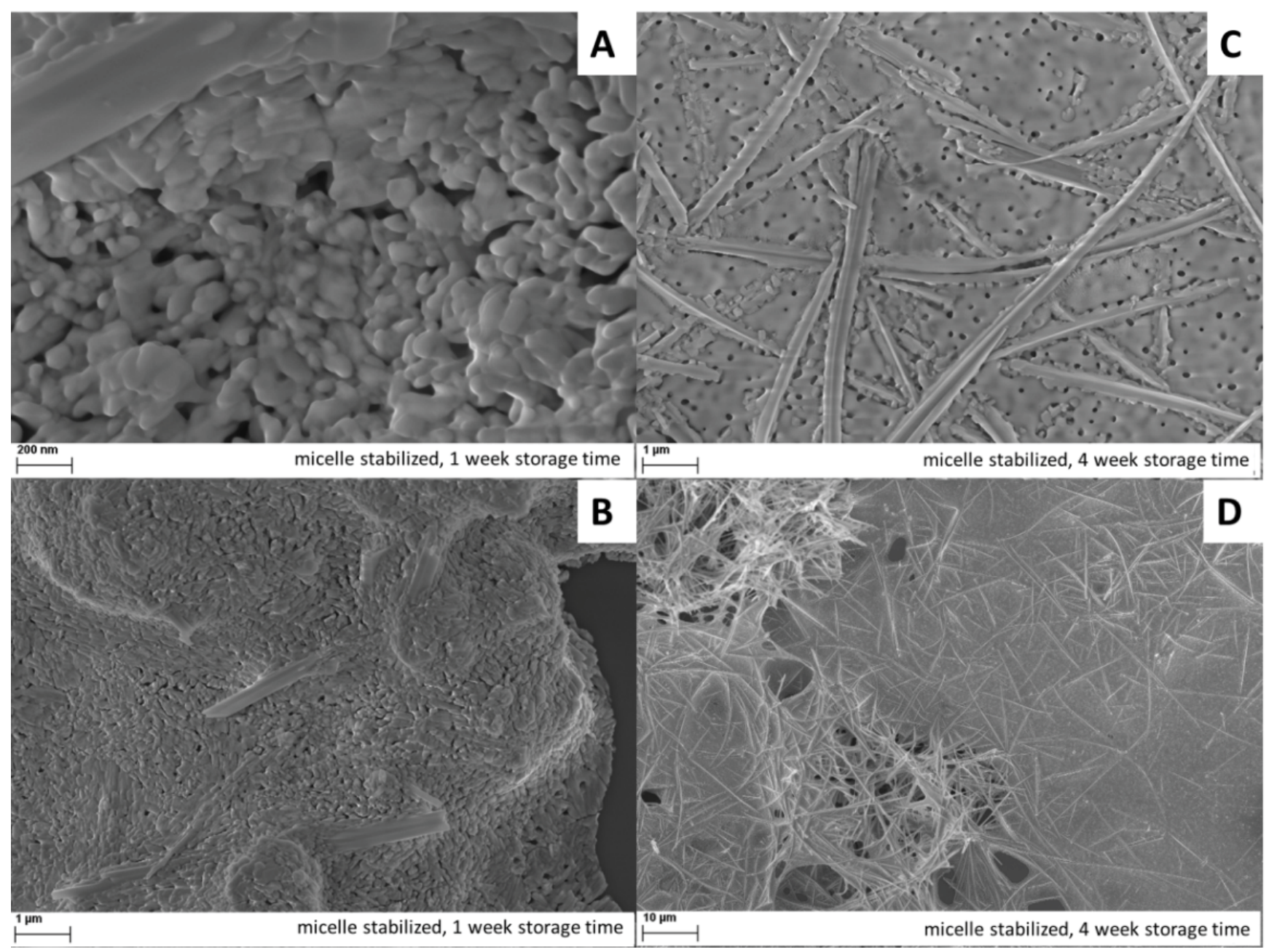

Figure 5: SEM pictures of Se particles, stabilized with TX 100 after 1 week $(A, B)$ and 4 weeks $(C, D)$ of storage. The particles are very spattered and form needlelike Se structures. After 4 weeks of storage, the particle size increased by one order of magnitude.

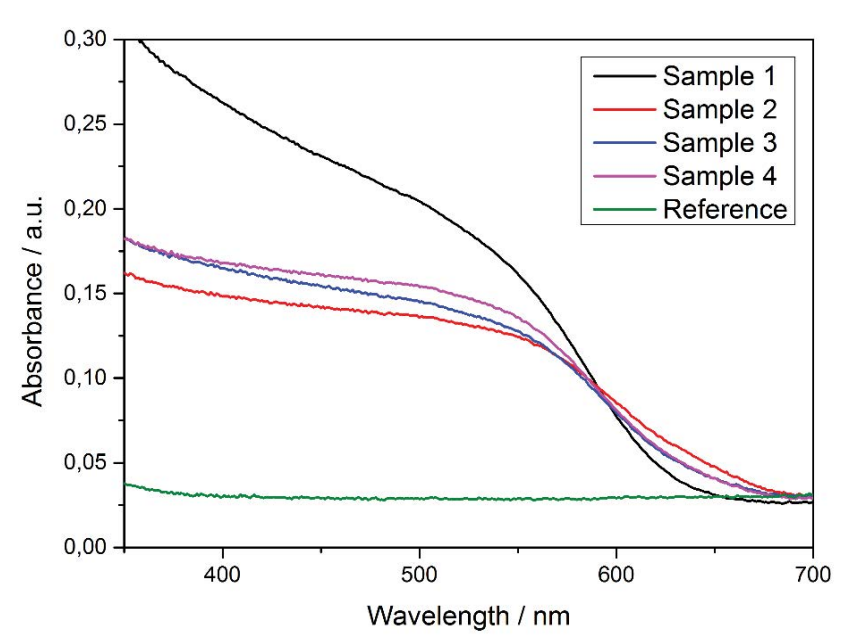

Figure 6: UV/VIS measurements showing the absorbance of the freshly prepared samples 1-4 and a reference sample without Se nanoparticles measured in an integrating sphere.

Apart from sample 1, the ratios are relatively constant, although sample preparations and syntheses were the same. In conclusion, we have a theoretical Se input of $250.00 \mathrm{mg}$ and a mean particle outtake of $102.21 \mathrm{mg}$, leading to a mean efficiency for the synthesis of $41 \%$. This value is rather low since there was no obvious loss of particles during the preparation. It could result from an incomplete conversion of selenium into particles. This aspect is due for further investigation and research, for the synthesis to become more efficient.

After determination of the concentration the transmission was measured using UV/VIS spectroscopy inside an integrating sphere. Figure 6 shows the absorbance of samples 1-4 and a reference sample that contained no Se nanoparticles.

The absorbance of sample 1 is significantly higher than the other three which was expected due to the higher amount of Se. Sample 2 shows less absorbance than sample 3 , although it has a larger amount of Se in dispersion. The reference sample without Se nanoparticles does not show any influence on absorbance.

From the shape of the UV/VIS spectra it is possible to draw conclusions about the particle size as smaller particles show a steeper curve and more total absorbance in the shown wavelength range than larger particles [26]. Sample 2 therefore has slightly larger particles than the others, sample 1 has the smallest particles. However all samples were freshly synthesized and are expected to show a particle size distribution similar to the one seen in Figure 3 (PVP, fresh) with a particle size of ca. $80 \mathrm{~nm}$. Depending on the time that passed between synthesis and measurement, the size distribution had slightly changed, and interferes with the concentration dependence, especially when the concentration is only changed a little bit. Larger particles or agglomerates appear as a lower concentrated dispersion than smaller and dispersed particles. 
Table 2: Calculated values of the concentrations c, extinction $E$ at $400 \mathrm{~nm}$ and extinction coefficient of Se at $400 \mathrm{~nm}$.

\begin{tabular}{|l|l|l|l|l|l|}
\hline Sample & $\mathrm{c}(\mathrm{Se}) / \times 10^{-4} \mathrm{~mol} / \mathrm{l}$ & $\mathrm{c}(\mathrm{Se}-\mathrm{NP}) / \times 10^{-9} \mathrm{~mol} / \mathrm{I}$ & $\mathrm{E}(400 \mathrm{~nm})$ & $\varepsilon(\mathrm{Se}, 400 \mathrm{~nm}) / I \cdot(\mathrm{mol} \cdot \mathrm{cm})^{-1}$ & $\varepsilon(\mathrm{Se}-\mathrm{NP}, 400 \mathrm{~nm}) / \mathrm{I} \cdot(\mathrm{mol} \cdot \mathrm{cm})^{-1}$ \\
\hline 1 & 1.78 & 9.69 & 0.262 & $1.47 \times 10^{3}$ & $2.70 \times 10^{7}$ \\
\hline 2 & 1.13 & 6.16 & 0.148 & $1.31 \times 10^{3}$ & $2.40 \times 10^{7}$ \\
\hline 3 & 1.01 & 5.52 & 0.165 & $1.63 \times 10^{3}$ & $2.99 \times 10^{7}$ \\
\hline
\end{tabular}

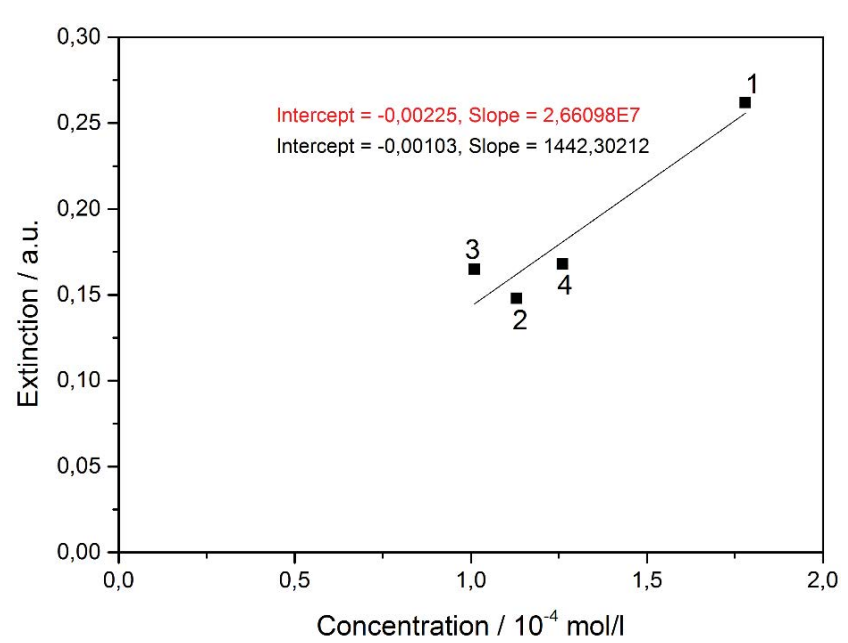

Figure 7: Extinction as a function of concentration for samples 3, 2, 4, 1 (from left to right). The slope of the linear fit-function visualizes the molar extinction coefficient. Fit function black for c (Se), red for c (Se-NP) (x-Axis not shown).

The molar extinction coefficient $\varepsilon$ of colloidal Se at this specific wavelength is calculated (Table 2) by taking the concentration of each dispersion and the respective extinction at a wavelength of $400 \mathrm{~nm}$ and insert all values into the Lambert-Beer law (equation 2). Moreover the amount of substance of the selenium colloid ( $80 \mathrm{~nm}$ diameter) was calculated for each sample to determine the extinction coefficient of the colloids. The sample thickness was the standard $10 \mathrm{~mm}$ cuvette.

The calculations lead to an average value for $\varepsilon(\mathrm{Se})$ of $1.44 \times 10^{3} \mathrm{l} /$ $(\mathrm{mol} \cdot \mathrm{cm})$ respectively $2.63 \times 10^{7} \mathrm{l} /(\mathrm{mol} \cdot \mathrm{cm})$ for $\varepsilon(\mathrm{Se}-\mathrm{NP})$ with a standard deviation error of $9 \%$. A plot of extinction $\mathrm{E}$ and concentration c, when a linear fit function is applied, (Figure 7) shows a straight line in compliance with Lambert-Beer law (eq. 1) with the slope visualizing the molar extinction coefficient. The values of $1.44 \times 10^{3}$ respectively $2.66 \times 10^{7}$ fit very well to the calculated average and the fit passes through the origin, thus satisfying the Lambert-Beer law.

The deviation error could result from measurement errors in the UV/VIS spectrometer and/or impurities in the samples. Another source of error could be the fact, that the stabilizer is still attached to the particles and also causes measurements errors. Reference measurements were recorded, showing no substantial influence on the UV/VIS spectra. But the polymers and micelles in absence of Se nanoparticles could behave differently from their counterparts attached to Se-NP. Moreover the particle size plays an important role for the UV/VIS measurements, showing a blue shift of the spectra for smaller particles [26]. As discussed above particle size and concentration both influence the UV/VIS spectra. Larger particles or agglomerates appear as a lower concentrated dispersion than smaller and dispersed particles. Therefore it is advised to keep the time between synthesis and measurement constant, as well as all the other synthesis parameters, so that the particle size distribution is well controlled and the change is comparable for all further samples. The standard deviation $9 \%$ obtained in these experiments contains the issues of interference between particle-size, agglomeration and dispersion concentration existing in the present work.

\section{Conclusions}

Se nanoparticles were synthesized by preparing a solution of $\mathrm{Na}_{2} \mathrm{SeSO}_{3}$ and then precipitated from that batch solution using $\mathrm{HCl}$. The Se nanoparticles were stabilized with PVP and TX100, which led to well dispersed suspensions. DLS measurements showed that both approaches generated particle sizes of about $80-100 \mathrm{~nm}$ with relatively low size distribution. Additionally, DLS revealed a high Zeta potential for both stabilization approaches of $>35 \mathrm{mV}$ indicating a good stability of the dispersions. However, compared to TX100 stabilized particles, PVP stabilized particles showed a better stability with almost no change in size and Zeta potential during a storage period of 4 weeks. The TX100 samples destabilized during this aging period, resulting in the loss of Zeta potential and leading to bigger particles of around $450 \mathrm{~nm}$. The stability and size of the particles were also investigated by taking SEM images of freshly prepared samples and after four weeks of storage. Stabilization with PVP led to stable spherical particles while stabilization with TX100 led to rather spattered, less stable particles which had a tendency of coagulation and formation of Se needles.

For any further processing of Se nanoparticles, especially for application as Se source for printable CIS absorbers, it is necessary to know the concentration of the dispersion. A new and rather simple approach of determining the concentration by applying UV/ VIS spectrometry and Lambert-Beer's law has been developed. EDX measurements have been conducted to measure the concentration in different Se dispersions. By measuring the transmission of these samples, the Lambert-Beer law was applied to determine the molar extinction coefficient of Se, which was calculated to be $1.44 \times 10^{3} \mathrm{l} /$ $(\mathrm{mol} \cdot \mathrm{cm})$ for $\varepsilon(\mathrm{Se})$ respectively $2.63 \times 10^{7} \mathrm{l} /(\mathrm{mol} \cdot \mathrm{cm})$ for $\varepsilon(\mathrm{Se}-\mathrm{NP})$ $\pm 9 \%$ in both cases. This allows to determine the Se concentration in dispersions in a very quick and simple fashion. It herewith allows to adjust the exact desired ratios in mixtures of Se with other particles or different dispersions. The experiments also revealed that the described synthesis has an efficiency of ca. $40 \%$.

\section{Acknowledgements}

The authors would like to thank Deutsche For schulungsgemeinschaft under contract number GRK 1161 and the Energy Campus Nürnberg (EnCN) for financial support.

\section{References}

1. Gilleo MA (1951) Optical Absorption and Photoconductivity of Amorphous and Hexagonal Selenium. J Chem. Phys 19: 1291.

2. Luo P, Yu P, Zuo R, Jin J, Ding Y, et al. (2010) The preparation of CulnSe2 films by solvothermal route and non-vacuum spin-coating process. Phys $B$ Condens Matter 405: 3294-3298.

3. Mehta SK, Chaudhary S, Kumar S, Bhasin KK, Torigoe K, et al. (2008) Surfactant assisted synthesis and spectroscopic characterization of selenium nanoparticles in ambient conditions. Nanotechnology: 19.

4. Yan Y, Noufi R, Al-Jassim M (2006) Grain-Boundary Physics in Polycrystalline CulnSe2 Revisited: Experiment and Theory. Phys RevLett 26: 205501

5. Möckel SA, Hölzing A, Hock R, Wellmann PJ (2013) In-situ phase formation study of copper indium diselenide absorber layers from Culn nanoparticles and evaporated selenium. Thin Solid Films 535: 133-137.

6. Contreras MA, Egaas B, Ramanathan K, Hiltner J, Swartzlander A, et al. (1999) Progress Toward $20 \%$ E ciency in $\mathrm{Cu}(\mathrm{In}, \mathrm{Ga}) \mathrm{Se} 2$ Polycrystalline ThinFilm Solar Cells. ProgPhotovoltaics Res 7: 311-316. 
7. Victor P, Nagaraju J, Krupanidhi SB (2000) Pulsed excimer laser ablated copper indium diselenide thin Films. Solid State Commun 116: 649-653.

8. Jiang F, Feng J (2006) Effect of temperature on selenization process of metallic Cu- In alloy precursors. Thin Solid Films 515: 1950-1955.

9. Kapur V K, Bansal A, Le P, Asensio OI (2003) Non-vacuum processing of Culn1- xGaxSe2 solar cells on rigid and flexible substrates using nanoparticle precursor inks. Thin Solid Films 431: 53-57.

10. Tripathi K, Bahishti AA, Majeed Khan MA, Husain M, Zulfequar M (2009) Optical properties of selenium - tellurium nanostructured thin film grown by thermal evaporation. Phys B CondensMatter 404: 2134-2137.

11. Zoppi G, Forbes I, Miles RW, Dale PJ, Scragg JJ, et al. (2009) Cu 2 ZnSnSe 4 thin film solar cells produced by selenisation of magnetron sputtered precursors. Prog Photovoltaics Res 17: 315-319.

12. Cheung JT, Sankur H (1988) Growth of thin films by laser-induced evaporation. Crit Rev Solid State Mater 15: 63-109.

13. Nandhakumar I, Elliott JM, Attard GS (2001) Electrodeposition of Nanostructured Mesoporous Selenium Films ( H I -eSe ). Chem Mater 13: 3840-3842.

14. Santos MC, Machado SAS (2004) Microgravimetric, rotating ring-disc and voltammetric studies of the underpotential deposition of selenium on polycrystalline platinum electrodes. J Electroanal Chem 567: 203-210.

15. Yoon S, Yoon T, Lee KS, Yoon S, Ha JM, et al. (2009) Nanoparticle -based approach for the formation of CIS solar cells. Sol Energy Mater Sol Cells 93: 783-788.

16. Möckel SA, Wernicke T, Arzig M, Köder P, Brandl M, et al. (2014) Low Temperature Formation of CulnSe 2 Solar Cell Absorbers by an All Printed Two Species Nanoparticulate Se + Cu - In Precursor. Thin Solid Films.
17. Ingole A, Thakare S, Khati N (2010) Green synthesis of selenium nanoparticles under ambient condition. Chalcogenide Lett 7: 485-489.

18. Stroyuk AL, Raevskaya AE, Kuchmiy SY, Dzhagan VM, Zahn DRT, et al. (2008) Structural and optical characterization of colloidal Se nanoparticles prepared via the acidic decomposition of sodium selenosulfate. Colloids Surfaces A Physicochem Eng 320: 169-174.

19. Shah CP, Kumar M, Bajaj PN (2007) Acid-induced synthesis of polyvinyl alcohol-stabilized selenium nanoparticles. Nanotechnology 18: 385607.

20. Zhang SY, Zhang J, Wang HY, Chen HY (2004) Synthesis of selenium nanoparticles in the presence of polysaccharides. Mater Lett 58: 2590-2594.

21. Nath S, Ghosh SK, Panigahi S, Thundat T, Pal T (2004) Synthesis of Selenium Nanoparticle and Its Photocatalytic Application for Decolorization of Methylene Blue under UV Irradiation. Langmuir 20: 7880-7883.

22. Schuster M, Möckel SA, Wibowo RA, Hock R, Wellmann (2013) Optimising The Parameters For The Synthesis Of Cuin-Nanoparticles By Chemical Reduction Method For Chalcopyrite Thin Film Precursors. MRS Proc 1538: 203-208.

23. Zhang Z, Zhao B, Lhu (1996) PVP Protective Mechanism of Ultrafine Silver Powder Synthesized by Chemical Reduction Processes. J Solid State Chem 121: 105-110.

24. Sidorov S, Bronstein L, Valetsky P, Hartmann J, Cölfen H, et al. (1999) Stabilization of Metal Nanoparticles in Aqueous Medium by PolyethyleneoxidePolyethyleneimine Block Copolymers. J Colloid Interface Sci 212: 197-211.

25. Shin HS, Yang HJ, Bin Kim S, Lee MS (2004) Mechanism of growth of colloidal silver nanoparticles stabilized by polyvinyl pyrrolidone in gammairradiated silver nitrate solution. J Colloid Interface Sci 274: 89-94.

26. Zin L, Chris Wang C (2005) Evidence on the size- dependent absorption spectral evolution of selenium nanoparticles. Materials Chemistry and Physics 92: 591-594. 